\title{
The IL-21 receptor augments Th2 effector function and alternative macrophage activation
}

\author{
John Pesce, ${ }^{1}$ Mallika Kaviratne, ${ }^{1}$ Thirumalai R. Ramalingam, ${ }^{1}$ \\ Robert W. Thompson, ${ }^{1}$ Joseph F. Urban Jr., ${ }^{2}$ Allen W. Cheever, ${ }^{3}$ \\ Deborah A. Young, ${ }^{4}$ Mary Collins, ${ }^{4}$ Michael J. Grusby, ${ }^{5}$ and Thomas A. Wynn ${ }^{1}$ \\ ${ }^{1}$ Immunopathogenesis Section, Laboratory of Parasitic Diseases, National Institute of Allergy and Infectious Diseases (NIAID), \\ National Institutes of Health, Bethesda, Maryland, USA. 2Nutrient Requirements \& Functions Laboratory, \\ Beltsville Human Nutrition Research Center, Agricultural Research Service, United States Department of Agriculture, Beltsville, Maryland, USA. \\ ${ }^{3}$ Biomedical Research Institute, Rockville, Maryland, USA. ${ }^{4}$ Wyeth Research, Cambridge, Massachusetts, USA. \\ ${ }^{5}$ Department of Immunology and Infectious Diseases, Harvard School of Public Health, Boston, Massachusetts, USA.
}

\begin{abstract}
The IL-21 receptor (IL-21R) shows significant homology with the IL-4R, and CD4+ ${ }^{+}$Th cells are an important source of IL-21. Here we examined whether the IL-21R regulates the development of Th2 responses in vivo. To do this, we infected IL-21 $\mathrm{R}^{-/-}$mice with the Th2-inducing pathogens Schistosoma mansoni and Nippostrongylus brasiliensis and examined the influence of IL-21R deficiency on the development of Th2-dependent pathology. We showed that granulomatous inflammation and liver fibrosis were significantly reduced in S. mansoniinfected IL-21 $\mathrm{R}^{-/-}$mice and in IL-21 $\mathrm{R}^{+/+}$mice treated with soluble IL-21R-Fc (sIL-21R-Fc). The impaired granulomatous response was also associated with a marked reduction in Th2 cytokine expression and function, as evidenced by the attenuated IL-4, IL-13, AMCase, Ym1, and FIZZ1 (also referred to as RELM $\alpha$ ) responses in the tissues. A similarly impaired Th2 response was observed following $N$. brasiliensis infection. In vitro, IL-21 significantly augmented IL-4R $\alpha$ and IL-13R $\alpha 1$ expression in macrophages, resulting in increased FIZZ1 mRNA and arginase-1 activity following stimulation with IL-4 and IL-13. As such, these data identify the IL-21R as an important amplifier of alternative macrophage activation. Collectively, these results illustrate an essential function for the IL-21R in the development of pathogen-induced Th2 responses, which may have relevance in therapies for both inflammatory and chronic fibrotic diseases.
\end{abstract}

\section{Introduction}

The IL-21 receptor (IL-21R) is a newly discovered member of the class I cytokine receptor family $(1,2)$. The receptor shows significant sequence and structural homology with the IL-4R $\alpha$ chain and is adjacent to the IL-4R $\alpha$ in the human and mouse genomes, while its ligand, IL-21, shares significant homology with the cytokines IL-2, IL-4, and IL-15 (3, 4). IL-21 and IL-21R are thus newly described members of the $\gamma$ chain-dependent cytokine network because of their homology with cytokines and receptors that require the $\gamma$ chain for functional signaling (5). Because all members of the $\gamma$ chain network exhibit important and unique roles in host immunity, there has been growing interest in dissecting the novel functions of the IL-21R during antigen-triggered immune responses in vivo.

Initial studies examining its function showed that IL-21 antagonizes NK cell expansion, yet it promotes antigen-specific T cell immunity including antitumor immunity (6-8), findings that suggested IL-21 can bridge innate and adaptive immune responses (9). IL-21 also regulates B cell and CD8 ${ }^{+} \mathrm{T}$ cell function in vivo (10-15). Additional studies suggested that IL- 21 is a Th 2 cytokine that can inhibit the differentiation of naive Th cells into IFN- $\gamma$-secreting

Nonstandard abbreviations used: AMCase, acidic chitinase; Arg-1, arginase-1; Con A, concanavalin A; FIZZ1, found in inflammatory zone-1; -R, receptor; s-, soluble; SEA, soluble egg antigen.

Conflict of interest: The authors have declared that no conflict of interest exists. Citation for this article: J. Clin. Invest. 116:2044-2055 (2006). doi:10.1172/JCI27727.
Th1 cells (16). Indeed, exogenous treatment with IL-21 potently inhibited IFN- $\gamma$ production without affecting other Th1/Th2associated cytokines, suggesting that the repression of IFN- $\gamma$ by IL-21 is highly specific. Thus, by virtue of its ability to suppress the development of Th1 cells, it was hypothesized that IL-21 might promote Th2 responses (16). Nevertheless, the involvement of the IL-21R signaling pathway in Th2 response development was not previously investigated in any Th2-dependent disorder.

In schistosomiasis, Th2 cytokines play an indispensable role in the pathogenesis of the disease $(17,18)$. Indeed, IL-4/IL-13-, IL-4R $\alpha-$, and Stat6-deficient mice all show significantly impaired granuloma formation and liver fibrosis following infection with Schistosoma mansoni (19-22). Given the recent classification of IL-2 1 as a Th2 cytokine $(16,23)$, the striking similarities between the IL-4 and IL-21 receptors $(3,4)$, and the critical role of the related IL-4R $\alpha /$ Stat 6 signaling pathway in this disease as well as in other Th2 cytokine-driven inflammatory disorders (24), an important question evolving from these studies was whether IL-21R signaling was playing a significant role in the initiation and/or maintenance of Th2 immunity.

To investigate the regulation and function of the IL-21R in vivo, several different experimental systems of Th2-dependent inflammation were examined, including models of pulmonary and hepatic inflammation as well as an experimental model of nematode infection $(18,25)$. In each case, the immune responses of WT animals were compared with IL-21R-deficient mice $(10,26)$. The data demonstrated that the development of Th2-associated pathology 
A

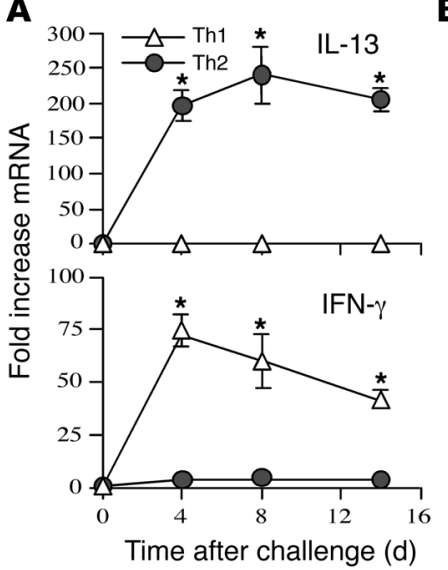

B

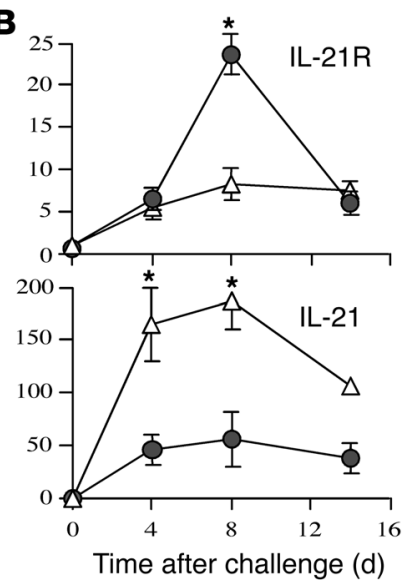

Figure 1

IL-21 and IL-21R expression during highly polarized Th1 and Th2

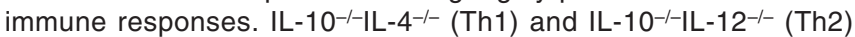
mice ( $n=5$ per group) were sensitized i.p. with freshly isolated eggs of S. mansoni and challenged i.v. 14 days later. On days 4, 8, and 14 after challenge, mice were sacrificed, and lung RNA specimens were prepared individually for real-time RT-PCR analysis of IL-13 and IFN- $\gamma$ (A) as well as IL-21 and IL-21R (B). Gene expression (mean \pm SEM) is expressed as the fold increase over unchallenged WT controls after normalization to HPRT. Similar results were obtained in several repeat experiments. ${ }^{*} P<0.05$.

was significantly reduced when IL-21 signaling was blocked. IL-21 also increased IL-4/IL-13 receptor expression and arginase activity in macrophages. The combined results from these studies revealed an important role for the IL-21R in Th2-driven inflammation and fibrosis. They also showed that IL-21 promoted the development of alternatively activated macrophages, thus providing a likely mechanism for the reduced Th2-dependent pathology in chronically infected IL-21 $\mathrm{R}^{-/-}$mice.

\section{Results}

Regulation of IL-21 and its receptor during Th1- and Th2-polarized responses. In comparison to IL-21 $(16,27)$, little is known about the regulation and function of the IL-21R. To determine whether IL-21 and its receptor are regulated during a pathological Th2 response in vivo, we used the S. mansoni model of granuloma formation, in which Th2 cytokines are known to play a prominent role in lesion formation (18). In initial studies, we sought to determine whether IL-21 and IL-21R mRNA expression was linked with polarized Th2 cytokine responses in vivo. To do this, we used mice that develop highly exaggerated Th1 (IL-4-/-IL-10-/-) or Th2 (IL-12-/-IL-10 $0^{-/-}$) cytokine responses following exposure to $S$. mansoni eggs. In IL-4-/IL-10-/- Th1 mice, IFN- $\gamma$ mRNA expression increased in the lung 75 -fold over baseline by day 4 after challenge and remained approximately 50-fold above background levels through day 14 (Figure 1A). IL-13 mRNA was not detectable in these mice at any time point, confirming the establishment of a highly polarized Th1 inflammatory response. In contrast, the IL-12-/-IL-10-/- Th2 mice displayed a 200- to 250-fold increase in IL-13 mRNA at all time points after challenge, with little to no change in IFN- $\gamma$. In contrast to the Th1/Th2 cytokines, which displayed a highly polarized pattern of expression, IL-21 was not associated with a polarized phenotype (Figure 1B). In both groups, IL-21 mRNA levels increased

at least 50-fold over baseline following challenge with schistosome eggs, although the increase observed in the Th1-polarized mice was on average 3- to 4-fold greater than the Th2-polarized animals (Figure 1B). IL-21R was also not specifically associated with a Th1 or Th2 immune response. However, in contrast to IL-21, which was more pronounced in Th1-skewed animals, the maximal response for the IL-21R was observed in the Th2-polarized mice.

Th2 cytokine production is reduced in the lungs of IL-21R-deficient mice. Based on the significant elevation in IL-21R expression in mice challenged with schistosome eggs (Figure 1), we next examined whether IL-21R signaling was influencing the development of the Th2 response. In these experiments, naive WT and IL-21R $\mathrm{R}^{-/-}$mice were injected i.v. with live schistosome eggs, and the production of Th2 cytokines and Th2-regulated genes was monitored in the lung, spleen, and draining lymph nodes over the following 14 days. In WT mice, IL-21R mRNA expression increased rapidly following egg exposure and remained elevated through day 14 after challenge (Figure 2A). IL-21 showed a similar profile, with peak expression occurring on day 7 and then declining slightly thereafter. Notably, there was a consistent and highly significant decrease in IL-21 expression on days 7 and 14 in the IL- $21 \mathrm{R}^{-/}$mice, suggesting that IL-21R was positively influencing the expression of its own ligand. Consistent with previous observations $(28,29)$, expression of the Th2-associated cytokines IL-4 and IL-13 rose gradually in the granulomatous tissues of WT mice, with 5- to 15fold increases detectable by day 14 . In contrast, there was a marked and significant decrease in IL- 4 and IL-13 mRNA expression in the IL-21 $\mathrm{R}^{-/-}$tissues. Although little change in IFN- $\gamma$ and IL-10 mRNA was detected in WT mice between days 4 and 14 after challenge, production of IFN- $\gamma$ and IL-10 also decreased slightly in the IL-21 $\mathrm{R}^{-1-}$ animals. Thus the reduced Th2 response observed in the IL-21 $\mathrm{R}^{-/-}$mice was not associated with increased Th1 cytokine production. In contrast to the granulomatous tissues, however, significant Th2 cytokine production was observed in lymph node and splenocyte cultures following in vitro stimulation with soluble egg antigen (SEA) or mitogen (concanavalin A [Con A]; Figure 2B). In fact, SEA consistently stimulated stronger IL-5, IL-10, and IL-13 responses in the lymph node cultures prepared from IL-21 $\mathrm{R}^{-/-}$mice. Nevertheless, consistent with the reduced Th2 response in the lung, a more rapid resolution of granuloma formation was observed in the IL-21 $\mathrm{R}^{-/-}$animals (Figure 2C). In addition, we observed a marked decrease in several genes associated with alternatively activated macrophages (30-33), providing further evidence of an overall reduction in the in vivo Th2 effector response in IL-21 $\mathrm{R}^{-}-$mice (Figure 2D).

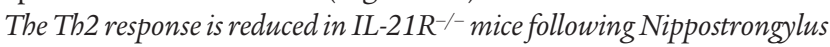
brasiliensis infection. To determine if the reduced Th2 effector response was specific to $S$. mansoni pulmonary granuloma formation, we infected WT and IL-21 $\mathrm{R}^{-/}$- mice with the intestinal nematode Nippostrongylus brasiliensis. Infection was established by inoculating third-stage larvae under the skin. As the parasites mature, they migrate from the site of inoculation and enter the lungs via the circulatory system. Once inside the lungs, the parasites trigger a vigorous and highly polarized Th2 response (34), which was confirmed by analyzing the expression of several Th2-associated genes in the lung (Figure 3A) and in lung-associated lymph nodes (Figure 3B). The lungs and lymph nodes of WT mice displayed marked increases in IL-4, IL-13, acidic chitinase (AMCase), found in inflammatory zone-1 (FIZZ1; also referred to as RELM $\alpha$ ), and Ym1 mRNA expression following N. brasiliensis infection (Figure 3, 
A
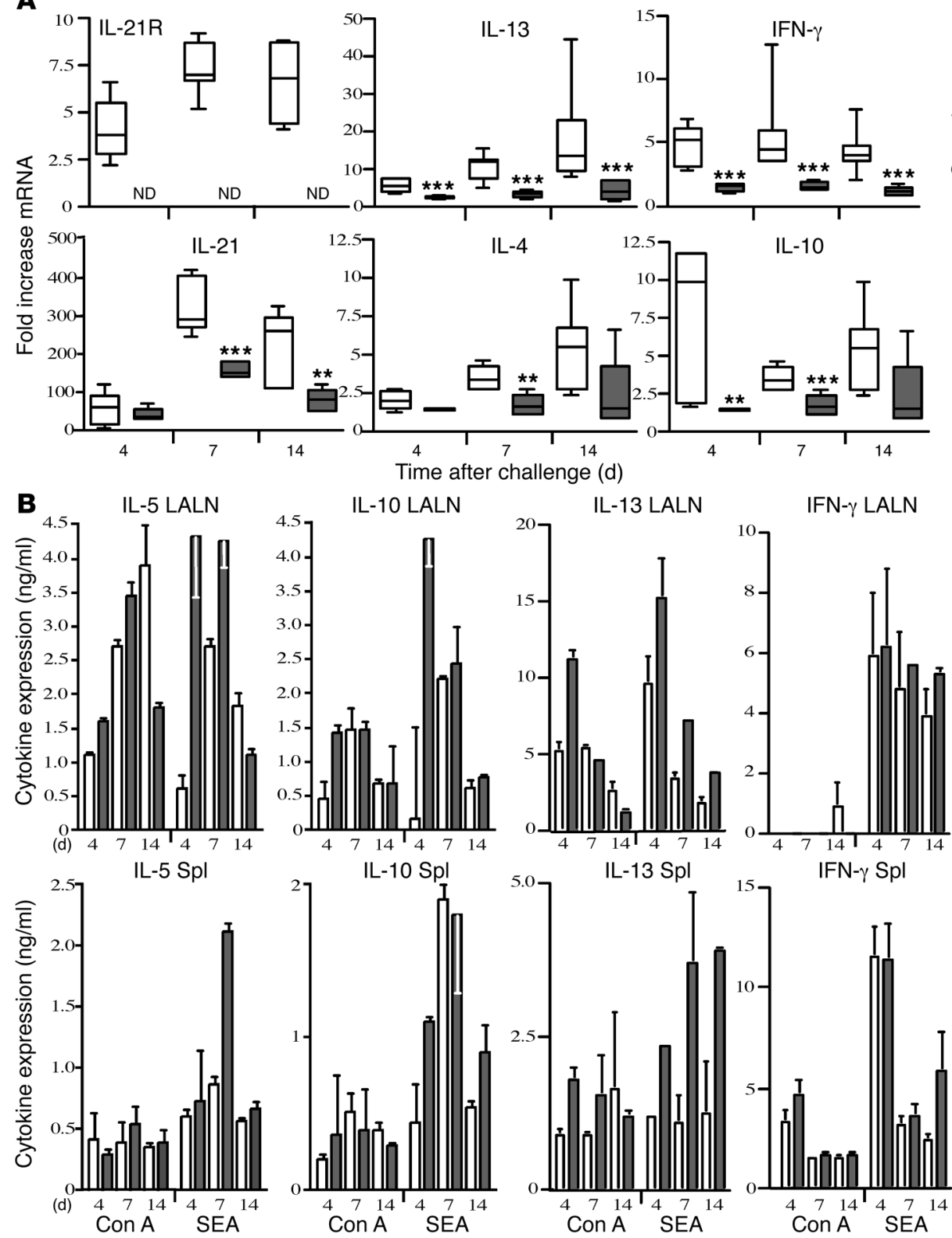
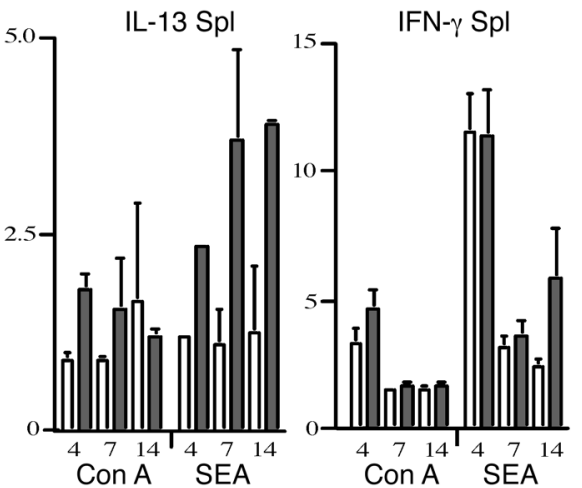
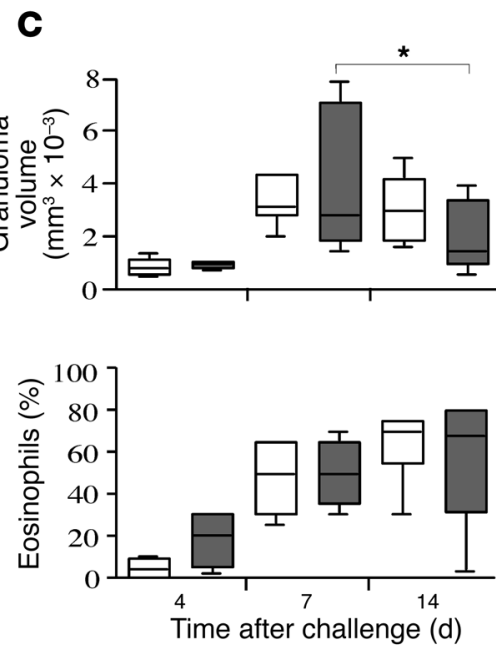

D

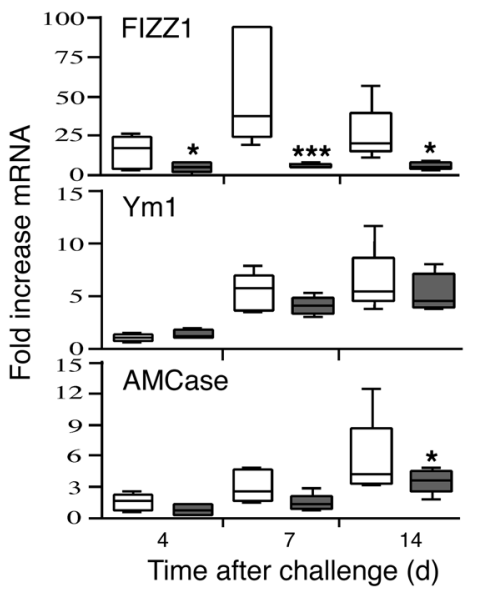

Figure 2

Th2 cytokine production is reduced in the lungs of schistosome egg-challenged IL-21R $\mathrm{R}^{-/}$mice. Groups of naive WT (white bars) and IL-21R ${ }^{-/}$ mice (gray bars) were i.v. challenged with live S. mansoni eggs and sacrificed on days 4, 7, and 14 after challenge. (A) RNA was prepared from lung tissues and analyzed individually ( $n=5$ per group/time point) by real-time RT-PCR. The top and bottom of the boxes indicate the seventy-fifth and twenty-fifth percentiles, respectively; the line within the box indicates the fiftieth percentile; and the top and bottom whiskers indicate the ninetieth and tenth percentiles, respectively, of the tested samples. ND, not detected. ${ }^{\star} P<0.05$, ${ }^{\star \star} P<0.01$, ${ }^{\star * \star} P<0.001$ versus WT. (B) Spleens (Spl) and lung-associated lymph nodes (LALN) were each pooled (2 separate groups, 3-4 mice per group), and single-cell suspensions were assayed for IL-5, IL-10, IL-13, and IFN- $\gamma$ after a 72-hour incubation in the presence of Con A (1 mg/ml) or SEA (20 mg/ml). Results are mean \pm SEM. Cytokines were below the level of detection in unstimulated cultures. (C) Granuloma size (volume, $\mathrm{mm}^{3} \times 10^{-3}$ ) and the percentage of eosinophils in granulomas were quantified microscopically. (D) Real-time PCR analysis of Th2-regulated inflammatory genes in granulomatous lung tissue. All data are representative of at least 2 separate experiments.

A and B). However, in agreement with the pulmonary granuloma model, we observed significantly reduced levels of IL-4, IL-13, and AMCase and slightly reduced levels of Ym1 and FIZZ1 mRNA in the lungs of the IL-21 $\mathrm{R}^{-/-}$mice (Figure $3 \mathrm{~A}$ ). The draining lymph nodes displayed a similar reduction, although the decreases in
Ym1 and FIZZ1 were more significant in the lymph nodes (Figure $3 \mathrm{~B}$ ). The only other major difference between the 2 tissues was the AMCase mRNA response, which appeared to be restricted to the lung. Together, these data confirm an important role for the IL-21R in Th2 response development in vivo. Notably, how- 
A
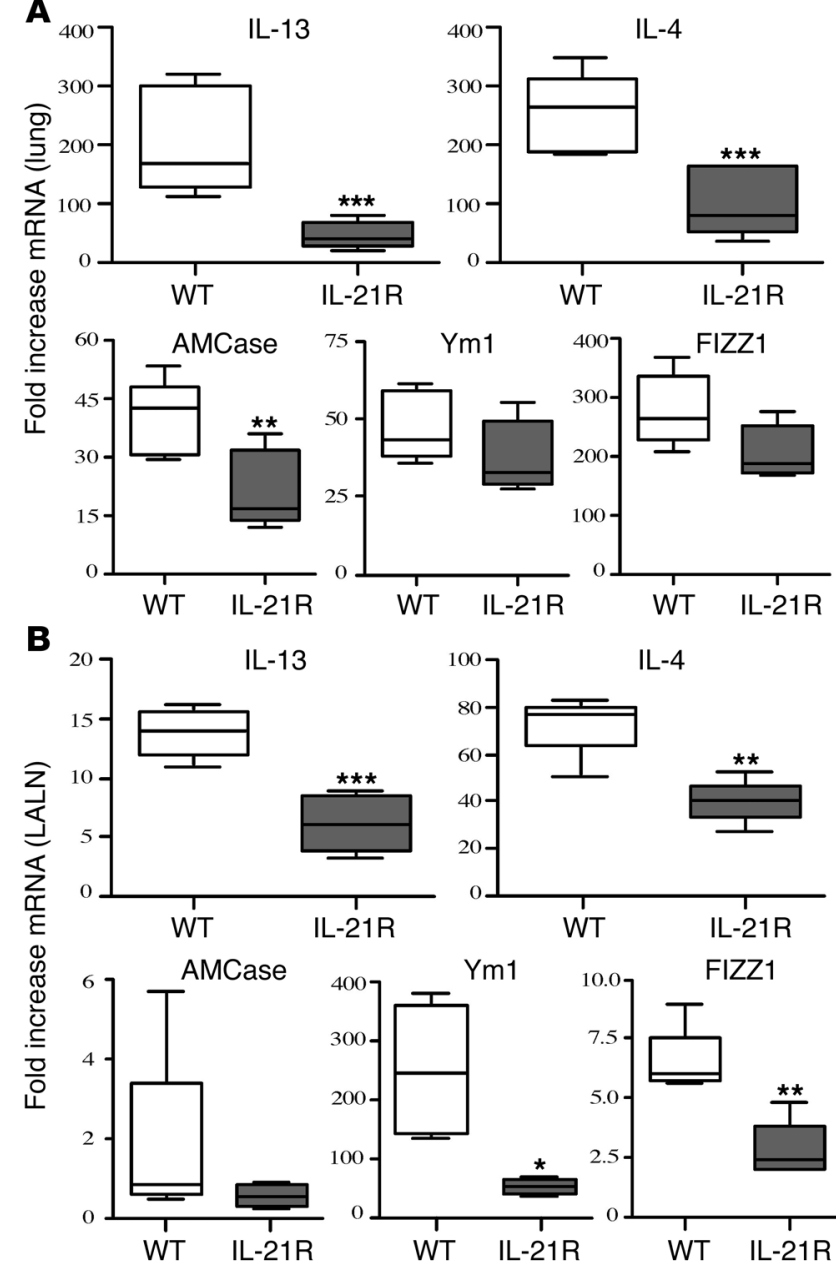

IL-4

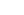


A
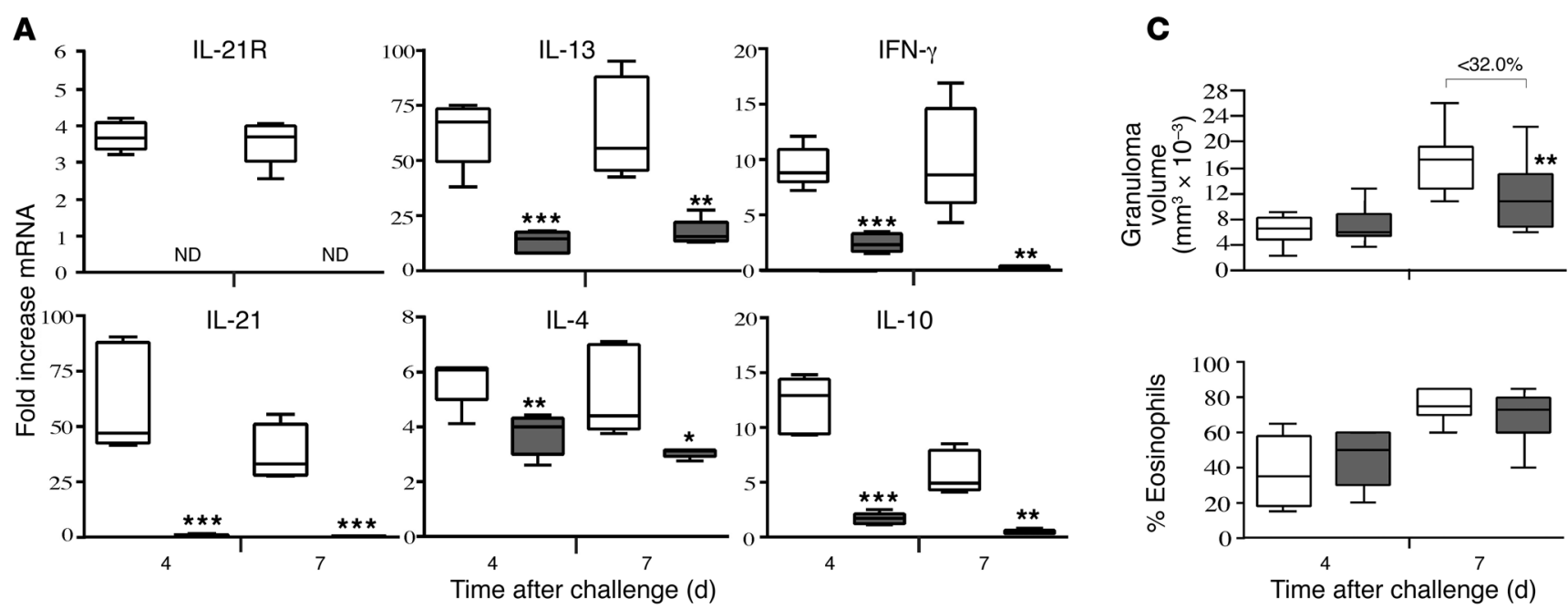

B
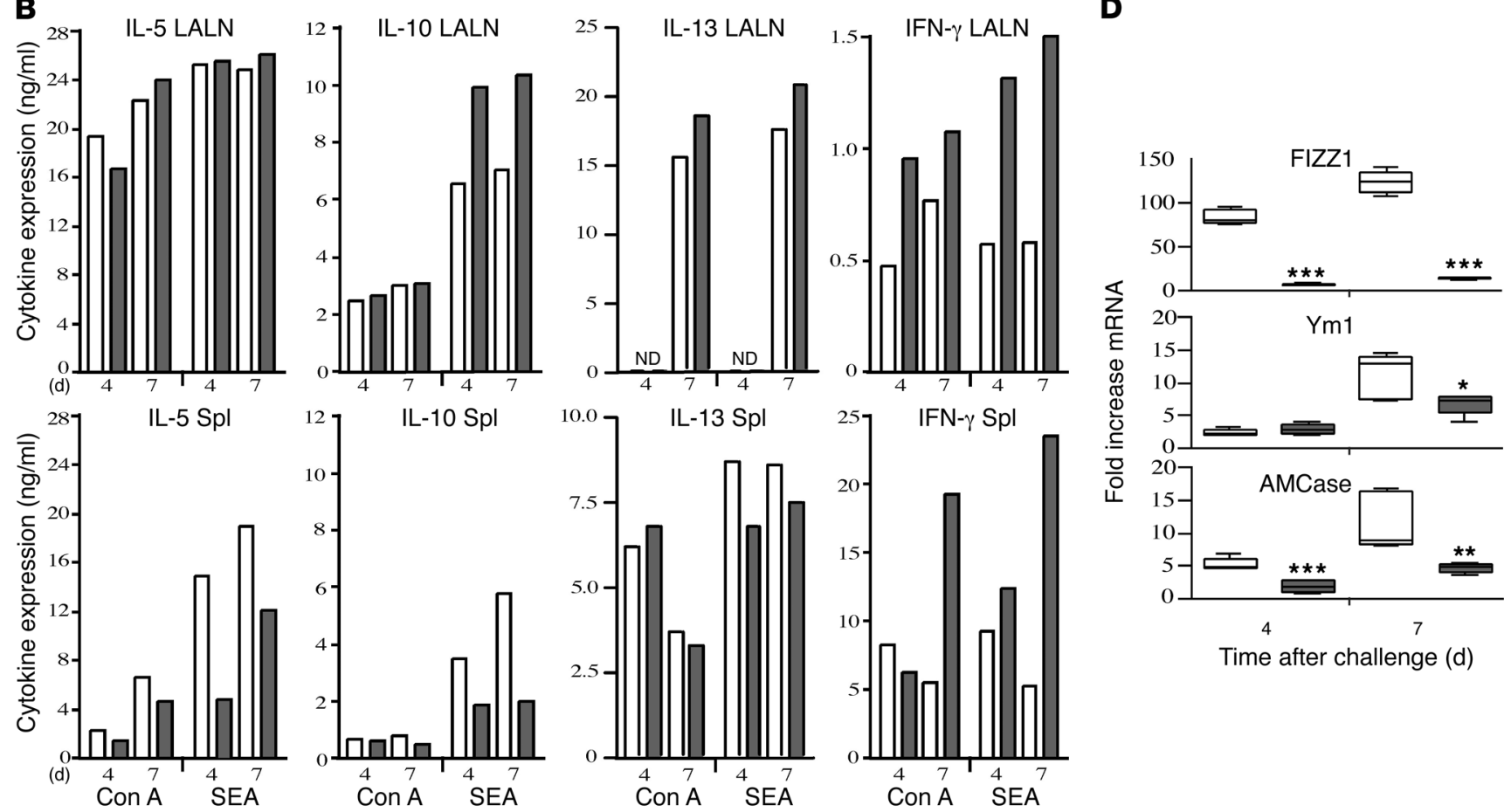

Figure 4

Th2 cytokine-driven inflammation is reduced in IL-21R $\mathrm{R}^{-1-}$ mice. WT (white bars) and IL-21 $\mathrm{R}^{-1-}$ mice (gray bars) were sensitized i.p. with eggs, challenged i.v. 2 weeks later with live $S$. mansoni eggs, and then sacrificed on days 4 and 7 after challenge. (A) RNA was prepared from lung tissues and analyzed individually ( $n=5$ per group/time point) by real-time RT-PCR as described above in the legend to Figure 2. (B) Spleens and lung-associated lymph nodes were assayed for IL-5, IL-10, IL-13, and IFN- $\gamma$ following antigen (SEA) or mitogen stimulation (Con A). (C) Granuloma size $\left(\mathrm{mm}^{3} \times 10^{-3}\right)$ and the percentage of eosinophils in granulomas were quantified microscopically in WT ( $n=10$ [day 4]; 15 [day 7]) and IL-21R ${ }^{-/}$mice $(n=11$ [day 4]; 16 [day 7]). (D) Real-time PCR analysis of Th2 inflammatory genes in granulomatous lung tissue $(n=5$ per group/time point). Data shown are the combined results of 3 separate experiments. ${ }^{*} P<0.05,{ }^{* *} P<0.01,{ }^{* *} P<0.001$ versus WT.

egg exposure; however, the pattern was similar in both WT and IL-21 $\mathrm{R}^{-/-}$mice (Figure 6B). Thus, changes in $\mathrm{CD}^{+} \mathrm{T}$ cell recruitment or expansion are unlikely to explain the decreased Th1/Th2 cytokine responses observed in the tissues. Instead, they appear to result from a more general reduction in the overall inflammatory response. Importantly, both groups effectively downmodulated their granulomatous responses by week 12 after infection (18). Consequently, there was no significant difference in granuloma size at the chronic time point (Figure 5C). We also detected minimal impairment in the Th2 cytokine response in the chronically infected knockout mice (Figure 5A). The marked reduction in FIZZ1 and $\mathrm{Ym} 1$ observed at the acute stage was also diminished in the chronically infected IL-21R $\mathrm{R}^{-/}$animals (Figure 5D). Nevertheless, expression of AMCase remained remarkably low at week 12 , suggesting a sustained diminution of at least a subset of the Th2-driven responses in chronically infected IL-21R $\mathrm{R}^{-/-}$mice. 
A

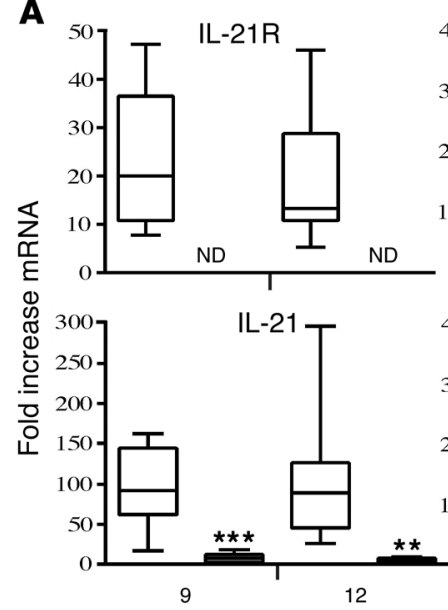

B

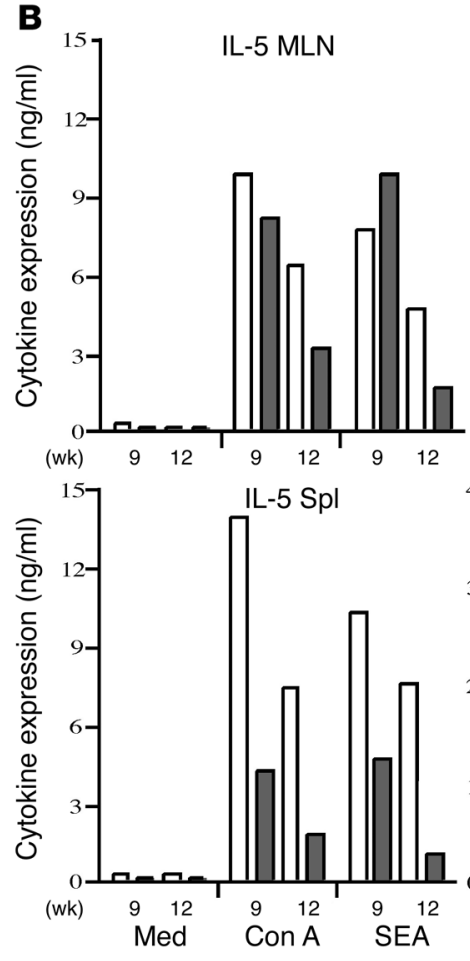

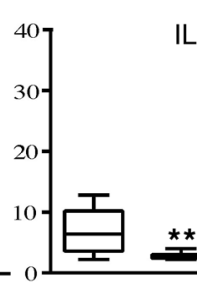

IL-13

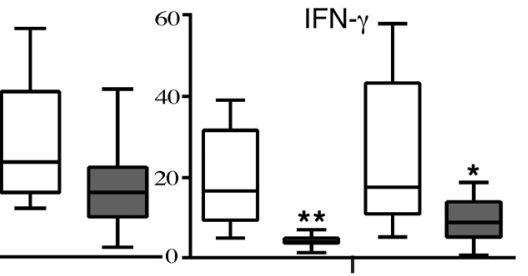

IL-10
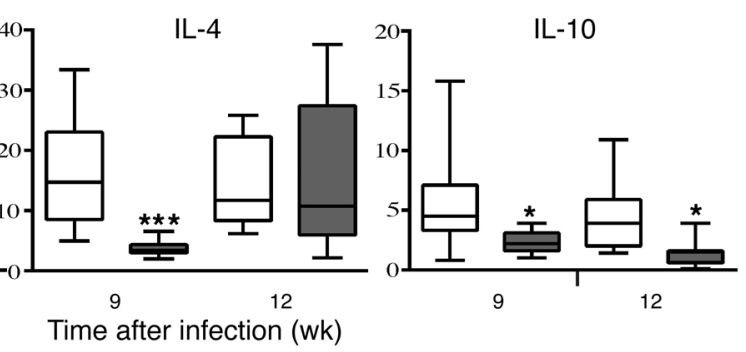

Time after infection (wk)
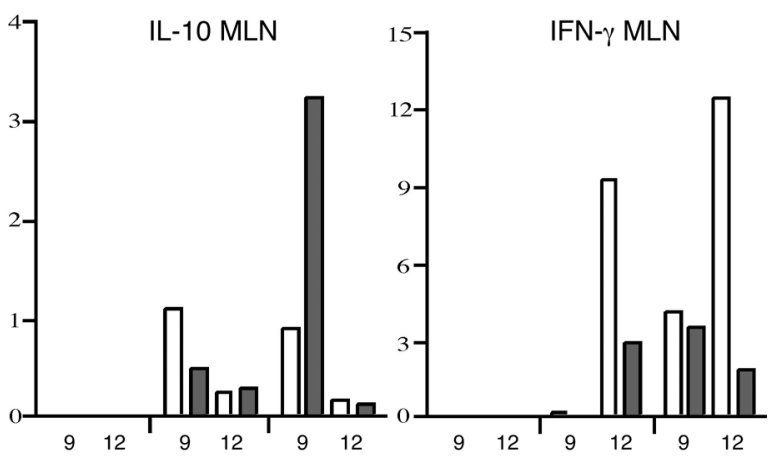

D
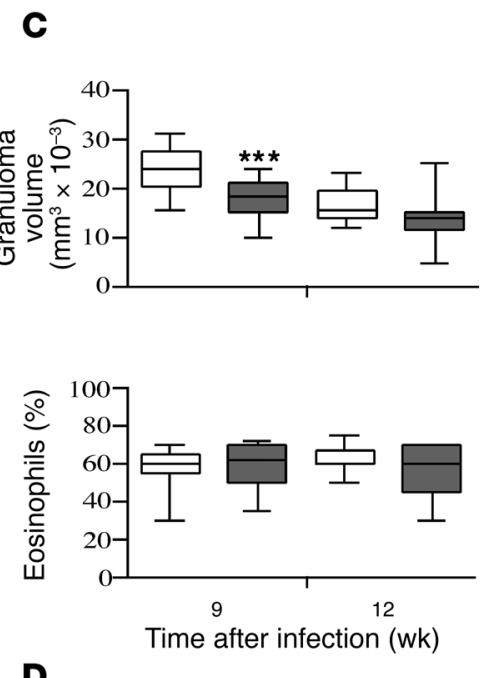
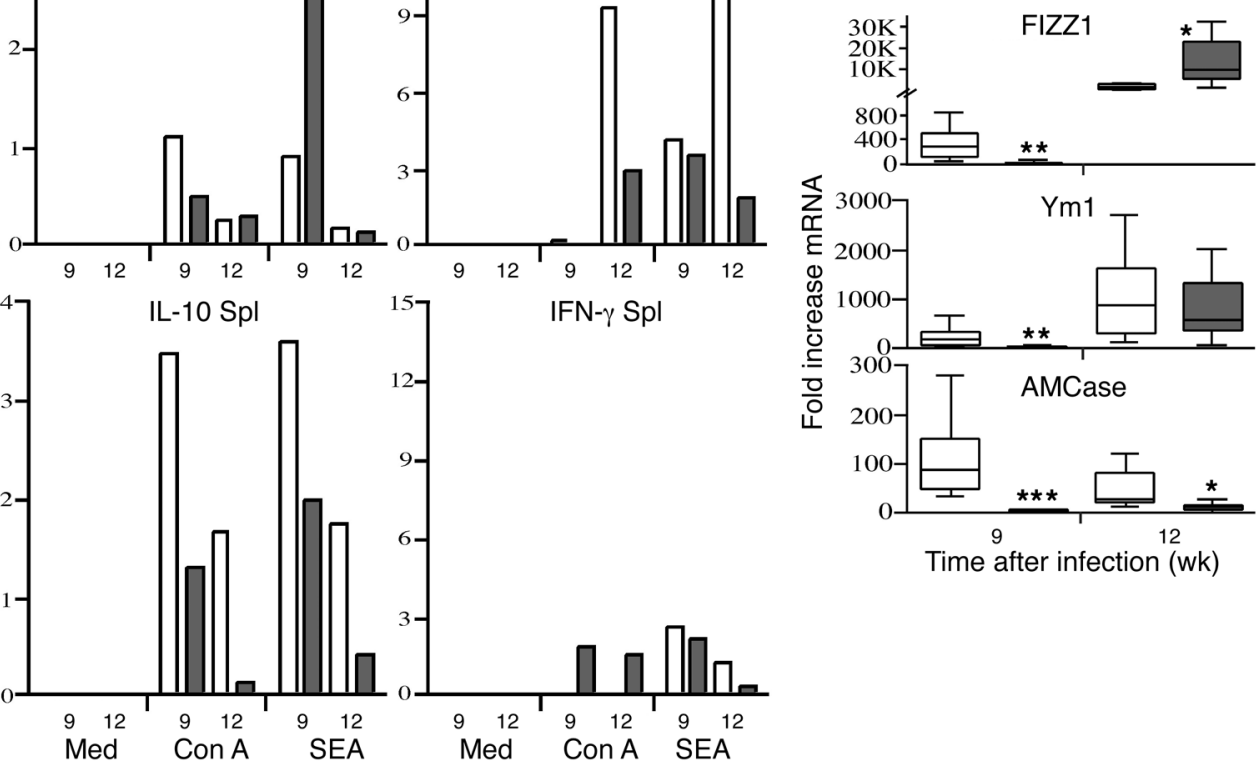

Figure 5

Chronic liver disease following percutaneous S. mansoni infection is reduced in the absence of IL-21R. WT (white bars) and IL-21R ${ }^{-/-}$mice (gray bars) were infected with 25-30 S. mansoni cercariae. All animals were sacrificed 9 (acute) or 12 (chronic) weeks after infection. (A) RNA was isolated from liver tissues and analyzed individually ( $n=8-10$ per group/time point) by real-time RT-PCR as described in the legend to Figure 2 . (B) Spleens and mesenteric lymph nodes (MLN) were pooled in groups of 2-4 mice, and single-cell suspensions were assayed for IL-5, IL-10, and IFN- $\gamma$. The data shown are the averages of 3 separate pooled groups. Med, medium alone. (C) Granuloma size $\left(\mathrm{mm}^{3} \times 10^{-3}\right)$ and the percentage of eosinophils in granulomas were evaluated microscopically in WT ( $n=30$ [week 9]; 17 [week 12]) and IL-21R ${ }^{--}$mice $(n=27$ [week 9]; 19 [week 12 ]). (D) Real-time PCR analysis of Th2 inflammatory genes in granulomatous liver tissue $(n=8-10$ per group/time point). Data shown are the combined results of 3 separate experiments conducted on week 9 and 2 performed on week 12 . ${ }^{\star} P<0.05$, ${ }^{\star \star} P<0.01,{ }^{\star * *} P<0.001$ versus WT.

The IL-21R $\mathrm{R}^{-/-}$mice were also examined for changes in serum Ablevels (Figure 7 and Table 1). Consistent with their suppressed cytokine responses (Figure 5A), the IL-21 $\mathrm{R}^{-/-}$mice displayed a marked reduction in parasite-specific $\operatorname{IgG}_{1}$ (Th2-associated $\mathrm{Ab}$ ) and $\mathrm{IgG}_{2 \mathrm{~b}}$ (Th1associated $\mathrm{Ab}$ ) titers, which was maintained at the chronic time point (Figure 7A). Interestingly, however, this was not accompanied by any significant change in IgE (Figure 7B), suggesting a selective impair- ment in only a subset of serum Ab isotypes. Exogenous IL-21 has been shown to inhibit IgE production (11), which may explain the slight elevation of IgE in the chronically infected IL-21R $\mathrm{R}^{-/-}$mice. Importantly, the overall reduction in Th2 responsiveness in the IL-21R $\mathrm{R}^{-/}$ mice was not attributed to differences in parasite burden since similar numbers of eggs and paired adult parasites were found in the tissues of both groups at all time points (Table 1). 
A

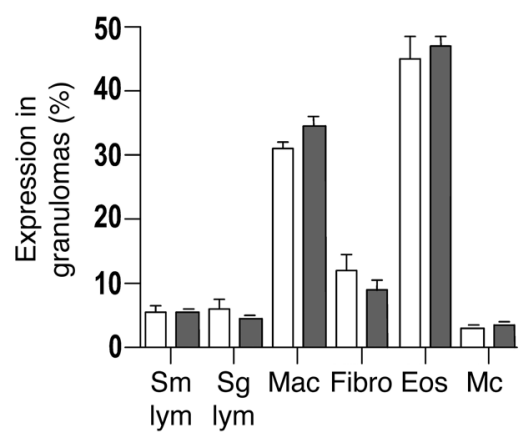

B

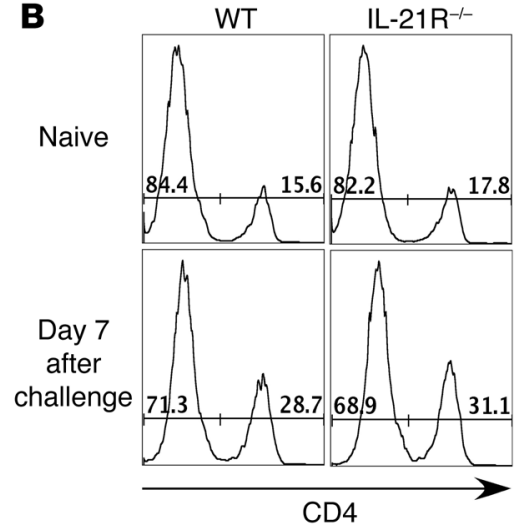

\section{Figure 6}

The cellular composition of granulomas is unchanged by IL-21R deficiency. (A) The cellular composition of individual granulomas was evaluated in the livers of 9 week infected WT (white bars; $n=10$ ) and IL-21R ${ }^{-1}$ (gray bars; $n=9$ ) mice. The mean \pm SEM of small lymphocytes (Sm lym; including the CD4/CD8 T cells), large lymphocytes (Lg lym; B cells and plasma cells), macrophages (Mac), fibroblasts (Fibro), eosinophils (Eos), and mast cells (Mc) are shown. (B) Lymphocytes were isolated from the perfused lungs of naive WT and $\mathrm{IL}-21 \mathrm{R}^{-/-}$mice and on day 7 following i.v. challenge with $5,000 \mathrm{~S}$. mansoni eggs. The numbers in the histograms indicate the percent of $\mathrm{CD}^{-}$and $\mathrm{CD} 4^{+} \mathrm{T}$ cells among total lung lymphocytes.
IL-21R deficiency slows the progression of liver fibrosis. Because Th2 cytokines are believed to play a major role in tissue fibrogenesis $(17,35)$, we examined the development and progression of hepatic fibrosis in S. mansoni-infected IL-21R $\mathrm{R}^{-/-}$mice. Liver hydroxyproline levels were assayed at various time points after infection as a direct measure of tissue collagen content. As expected, marked hepatic fibrosis was observed in the infected WT mice (Figure 7C). In contrast, the IL-21 $\mathrm{R}^{-/-}$mice displayed significantly less fibrosis at both the acute and the chronic time points. Notably, by week 29 after infection the IL-21R $\mathrm{R}^{-/-}$mice exhibited a greater than $50 \%$ decrease in total liver collagen content compared with WT mice (Figure 7D), thus confirming an important and indispensable role for the IL-21R in the progression of Th2-dependent fibrosis.

We also examined whether an IL-21 inhibitor could slow the progression of fibrosis in infected WT mice. For these experiments, groups of C57BL/ 6 mice were treated with soluble IL-21R-Fc (sIL-21R-Fc) or control protein for a total of 5 weeks, starting at week 6 after infection, around the time when eggs are first detected in the liver. Although both groups had similar worm and tissue egg burdens (data not shown), mice receiving the IL-21 blocker displayed a greater than $50 \%$ reduction in hepatic fibrosis at the termination of the experiment (Figure 7E). IL-4 and IL-13 mRNA expression also decreased in the liver, and granuloma size was reduced approximately $15 \%$ (data not shown). Thus the data nicely compliment the experiments performed with IL-21R $-1-$ mice.

IL-21 signaling promotes the development of alternatively activated macrophages. Because arginase- 1 (Arg- 1 ), FIZZ1, and TGF- $\beta 1$ have been linked with the development of fibrosis, and expression of several Th2/Stat6-regulated genes were reduced in the diseased tissues of IL-21R ${ }^{-1}$-mice (Figures 2-5) (30, 33), we examined whether Arg-1, FIZZ1, and TGF- $\beta 1$ were directly modulated in macrophages following stimulation with IL-21. Arg-1 and FIZZ1 are also wellknown markers of alternatively activated macrophages (33). For these studies, bone marrow-derived macrophage cultures were generated and then stimulated with various combinations of IL-4, IL-13, and IL-21. As expected, IL-4 and IL-13 both increased Arg1 and FIZZ1 mRNA expression, with an additive effect observed when the two stimuli were used in combination (Figure 8A). Notably, however, although IL-21 had no effect on either gene when used alone, cultures that were pretreated with IL-21 displayed highly significant increases in Arg-1 and FIZZ1 mRNA expression when subsequently stimulated with IL-4 and IL-13 (Figure 8A). The same combination also significantly increased the function of arginase in the cells, which was assessed by assaying the produc- tion of urea (Figure 8B). In contrast, IL-21 had no effect on the levels of total or active TGF- $\beta 1$ in the culture supernatants (Supplemental Figure 1; available online with this article; doi:10.1172/ JCI27727DS1). Unexpectedly, we found that IL-21 treatment alone significantly increased the expression of IL-4R $\alpha$ and IL-13R $\alpha 1$ (Figure 8C). In contrast, IL-4 and IL-13 had no effect when used alone (Figure 8C), and there was no additional effect when the three stimuli were used in combination (data not shown).

Because the IL-13R $\alpha 2$ can also influence IL-13-dependent signaling $(32,35,36)$, we also examined whether IL-21 regulates the production of the IL-13R $\alpha 2$. Not surprisingly, because the IL-13R $\alpha 2$ is primarily produced by nonhematopoietic cells like fibroblasts and smooth muscle $(19,38-40)$, we found no evidence of decoy receptor regulation in our bone marrow-derived macrophage cultures (data not shown). However, when we examined the regulation of the decoy receptor in vivo, IL-21 downregulated IL-13R $\alpha 2$ mRNA expression in the lungs of i.v. egg-challenged mice and significantly decreased the levels of the sIL-13R $\alpha 2$ in their serum (Figure 8D). Together these data suggest that IL-21 contributes to the development of alternatively activated macrophages by upregulating the Th2 IL-4R (signaling receptor) in macrophages and by simultaneously decreasing the levels of the sIL-13R $\alpha 2$ (decoy receptor) in the serum. Both mechanisms likely contributed to the increased activation of Arg-1 and FIZZ1 in the IL-4/IL-13-stimulated macrophages. As such, they provide an additional mechanistic explanation for the impaired Th2 responses and Th2-dependent fibrosis in the helminth-infected IL-21 $\mathrm{R}^{-/-}$mice.

\section{Discussion}

IL-21 was recently characterized as a Th2 cytokine that can inhibit the differentiation of naive Th cells into IFN- $\gamma$-producing Th1 cells (16). Because the immune response in schistosomiasis evolves from an early IFN- $\gamma$ to a sustained and dominant Th2 response (18), we examined the influence of IL-21R signaling on the development of helminth-induced Th2 responses. Infection of WT mice with S. mansoni increased IL-21 and IL-21R expression in the liver, confirming an association of IL-21 signaling with helminth-induced Th2 immunity. However, in the lung, schistosome eggs induced significant IL-21 expression during both Th1- and Th2-polarized responses. In fact, IL-21 expression increased the most when mice were polarized to a Th1 response. These data suggest that IL-21 exhibits a less restricted pattern of expression than that of the other Th2-associated cytokines. The receptor for IL-21 also failed to display a Th1/Th2-specific pattern. However, 
Table 1

Worm and egg burdens

$\begin{array}{llrrr}\begin{array}{l}\text { Week } \\ \text { after } \\ \text { infection }\end{array} & \text { Group } & \text { Worm pairs } & \text { Total worms } & \begin{array}{c}\text { Eggs per } \\ \text { worm pair } \\ \text { (in thousands) }\end{array} \\ 9 & \text { WT }(n=29) & 6.53 \pm 0.63 & 15.53 \pm 1.55 & 7.29 \pm 0.49 \\ 9 & \text { KO }(n=26) & 5.00 \pm 0.70 & 12.30 \pm 1.74 & 9.63 \pm 1.16 \\ 12 & \text { WT }(n=19) & 3.74 \pm 0.60 & 10.44 \pm 2.08 & 14.75 \pm 1.91 \\ 12 & \text { KO }(n=19) & 4.05 \pm 0.62 & 9.89 \pm 1.77 & 14.50 \pm 1.95\end{array}$

Values for each group are mean \pm SEM. No difference in infection intensity was noted in any experiment.

the IL-21R was induced nearly 4-fold more in the lungs of Th2than in Th1-polarized mice, which provided one of the first indications that IL-21R signaling might be involved in the regulation of Th2-mediated inflammation.

To determine whether Th2 effector responses were compromised in the absence of the IL-21R, we examined the expression of several genes that are induced preferentially under Th2-polarizing conditions. These genes included AMCase, Ym1, and FIZZ1, all of which are thought to play important and nonredundant roles in the regulation of Th2-mediated inflammation (30-32, $36,41)$. Although some variation was observed during a primary, secondary, or chronic immune response, in each case the IL-21 $\mathrm{R}^{-/-}$ mice displayed highly significant decreases in these Th2-associated genes. $\mathrm{Ym} 1$ and AMCase are members of a family of proteins that share homology with chitinases of lower organisms (30). Although their exact function in host immune reactions remains uncertain, they are thought to play important roles in eosinophil chemotaxis, tissue remodeling, and fibrosis. Indeed, a recent study showed that AMCase neutralization could ameliorate allergen-driven inflammation and airway hyperresponsiveness, thus suggesting the participation of mammalian chitinases in Th2 immunity (31). FIZZ1 is also associated with tissue fibrogenesis $(36,42)$. Consequently, a major function of the IL-21R may be to regulate the mechanisms of wound healing and fibrosis. Therefore, in addition to its participation in helminth-induced immune responses, the IL-21R may be involved in the regulation of a variety of Th2-mediated inflammatory disorders.

In schistosomiasis, IL-21R deficiency had a profound effect on the progression of the disease. Although infection intensities were the same in WT and IL-21 $\mathrm{R}^{-1}$ mice, the egg-induced inflammatory response decreased significantly in the absence of the IL-21R. We also observed a marked reduction in secondary granuloma formation and a faster resolution of primary granulomas in the lung. Together, these data illustrate an indispensable role for the IL-21R in granulomatous inflammation. As previous studies showed that IL-4 and IL-13 are essential for lesion formation (19), we therefore hypothesized that the IL-21R was either directly or indirectly affecting the activity of these cytokines. We knew that IL-21 was not acting alone, since extremely high levels of IL-21 were observed in IL-4/IL-10 double-knockout mice, yet granuloma formation was almost completely ablated in these Th2-deficient animals $(43,44)$. Thus, IL-21 appears to collaborate with IL-4 and IL- 13 to induce a maximal response. We also observed no detectable change in the cellular composition of the granulomas in IL-21 $\mathrm{R}^{-/-}$mice and no specific impairment in $\mathrm{CD} 4^{+} \mathrm{T}$ cell recruitment. Together, these findings suggested that the IL-21R regulates the development of parasite-induced pathology by modulating the overall intensity of the Th2 effector response.

IL-21 is not thought to regulate IL-4-induced Th2 cell differentiation directly $(11,16)$. Instead, it was hypothesized in a recent paper that IL-21 might amplify Th2-driven responses by downregulating the expansion of IFN- $\gamma$-producing Th1 cells (16). As such, we theorized that the IFN- $\gamma$ response in schistosome-infected mice might increase in the absence of the IL-21R. Although a small increase was observed in lung-associated lymph nodes in vitro, IFN- $\gamma$ production was consistently reduced in the granulomatous tissues. Thus, there was almost no evidence from our studies that the endogenous IL-21R was critically involved in the inhibition of IFN- $\gamma$ production during helminth infection. Instead, the IL-21 $\mathrm{R}^{-/-}$mice simultaneously generated weaker Th1 and Th2 cytokine responses in the tissues. The significant reduction in $\mathrm{IgG}_{2 \mathrm{~b}}$ (Th1-associated) and $\mathrm{IgG}_{1}$ (Th2-associated) $\mathrm{Ab}$ titers at all times after infection supports this conclusion. Th2 cytokines were also decreased at the mRNA level in both the lungs and the lymph nodes following $N$. brasiliensis infection. Indeed, all of the direct ex vivo data confirmed a marked reduction in Th2 cytokine expression and function within the affected tissues. Nevertheless, there was no consistent reduction in Th2 cytokine production by isolated lymphocytes following antigen restimulation, which suggests the $\mathrm{IL}-21 \mathrm{R}^{-/-}$mice are capable of generating significant Th2 responses, at least in vitro. We therefore conclude that the IL-21R is selectively augmenting Th2
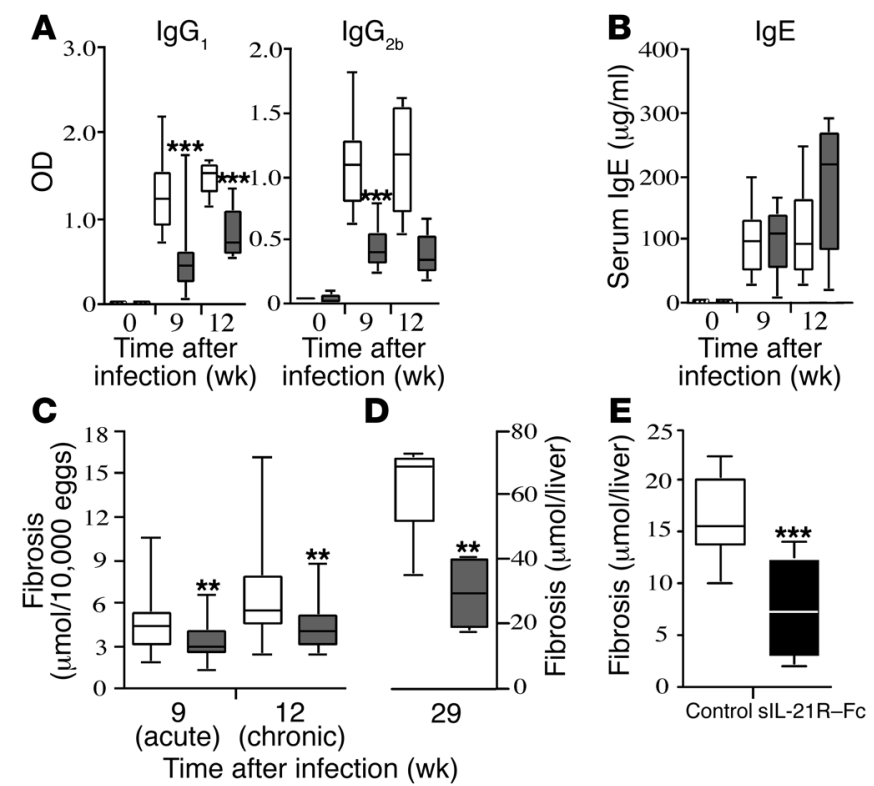

\section{Figure 7}

IL-21R deficiency significantly slows the progression of Th2 cytokinedependent fibrosis. WT (white bars) and IL-21R ${ }^{-/-}$mice (gray bars) were infected with $S$. mansoni cercariae. Animals were sacrificed 9 (acute) and 12 weeks (chronic; A-C) or 29 weeks (late chronic; D) after infection. (A) Mice were bled at the time of sacrifice, and SEA isotype-specific Ab titers were determined by ELISA. (B) Total serum IgE values. (C-E) Fibrosis was assessed by the amount of hydroxyproline ( $\mu \mathrm{mol}$ ) detected in the liver per 10,000 eggs (C) or per the total liver (D and $\mathbf{E}$ ). In $\mathbf{E}$, infected WT C57BL/6 mice were treated with either an $\operatorname{lgG}_{2 a}$ control Ab or with sIL-21R-Fc for 5 weeks. ${ }^{\star} P<0.05$, ${ }^{\star \star} P<0.01$, ${ }^{* * \star} P<0.001$ versus WT. 


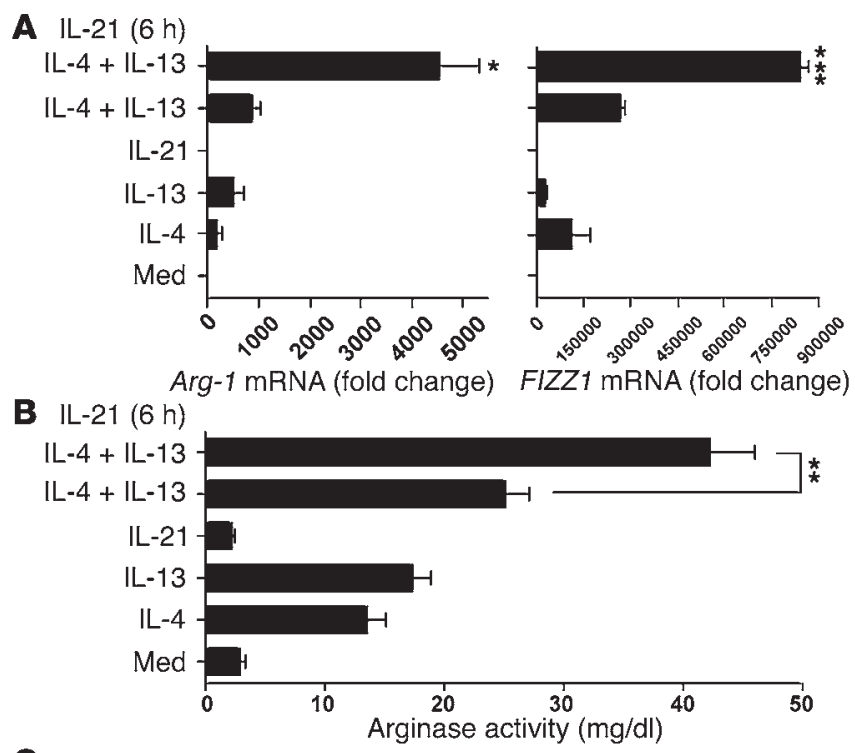

C
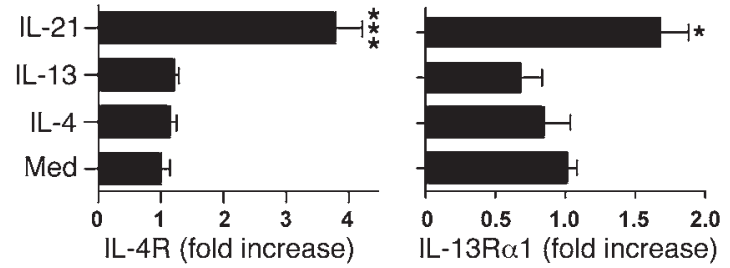

D

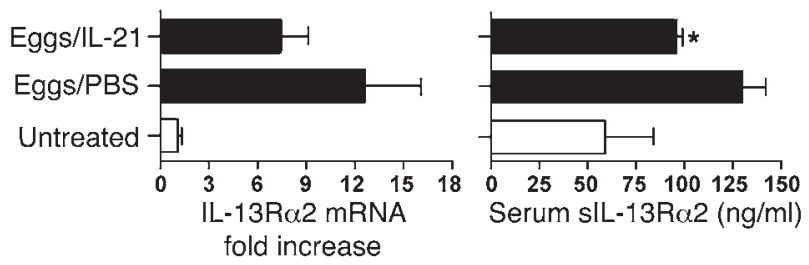

responses in vivo. In addition to promoting the Th2 response, the IL-21R also increased IL-21 production. Thus, the IL-21R appears to operate in an autocrine fashion to drive Th2 cytokine expression and Th2 effector functions in vivo.

To further elucidate the mechanisms involved, we also examined whether IL-21 was directly modulating macrophage function, because our in vivo data showed a marked reduction in several genes that have been associated with the "alternatively activated" phenotype $(33,45)$. Macrophages and fibroblasts exhibiting an alternatively activated phenotype are major cellular constituents of schistosome granulomas, and functional studies suggested they are critically involved in the progression of the disease (46). Indeed, an important study by Brombacher et al. showed that mice that are completely deficient in alternatively activated macrophages develop lethal egg-induced pathology following infection with $S$. mansoni (47). In addition, because macrophage-derived TGF- $\beta 1$ has been implicated in the mechanism of IL-13-mediated fibrosis $(48,49)$, we examined whether IL-21 was modulating TGF- $\beta 1$ production in macrophages. To investigate these issues, we measured Arg- 1 and FIZZ1 mRNA, arginase activity, and TGF- $\beta 1$ protein responses in bone marrow-derived macrophages following stimulation with various combinations of IL-21, IL-4, and IL-13. Arg-1 and FIZZ1 are IL-4R $\alpha /$ Stat6-dependent genes (42, 46, 50); therefore, they serve as functional markers of alternative macro-

\section{Figure 8}

IL-21 signaling promotes alternative macrophage activation by modulating IL-13R expression. Bone marrow-derived macrophages were treated with IL-21 $(20 \mathrm{ng} / \mathrm{ml})$, either alone or in combination with IL-4 $(20 \mathrm{ng} / \mathrm{ml})$ and IL-13 $(20 \mathrm{ng} / \mathrm{ml})$ overnight. In some experiments, macrophages were pretreated with IL-21 for 6 hours prior to IL-4 and IL-13 administration. Cells were lysed 20 hours later, and RNA was analyzed individually by real-time RT-PCR. (A) The ability of IL-21 to promote alternative macrophage activation was assessed by measuring Arg-1 and FIZZ1 mRNA expression. (B) Arginase activity was quantified in cell lysates by measuring the conversion of L-arginine to Urea $(\mathrm{mg} / \mathrm{dl} \pm \mathrm{SEM}$; triplicate measurements). (C) Expression of IL-4R $\alpha$ and IL-13R $\alpha 1$ mRNA was evaluated by real-time PCR. IL-13R $\alpha 2$ mRNA was nearly undetectable in all conditions (not shown). The data shown in A, B, and C are representative of 3 separate experiments. (D) Naive C57BL/6 mice were challenged i.v. with 5,000 live $S$. mansoni eggs and treated with PBS or recombinant IL-21 ( $2 \mu \mathrm{g} /$ dose) every other day from day 1 through day 6 . Animals ( $n=5$ per group) were sacrificed on day 7 , and lung IL-13R $\alpha 2$ mRNA levels were assayed by real-time PCR and expressed as fold increase over untreated controls. Mice were also bled at the time of sacrifice, and the amount of SIL-13R $\alpha 2$ in individual serum samples was assayed by ELISA. ${ }^{*} P<0.05$; ${ }^{* *} P<0.01$; ${ }^{* * *} P<0.001$. Similar results were obtained in a separate study.

phage activation. Importantly, the findings suggested that when macrophages are exposed to IL-21, they become much more sensitive to the Arg-1- and FIZZ1-inducing activities of IL-4 and IL-13. Arginase activity, as assessed by the production of urea, also increased significantly, confirming IL-21 as an important stimulus for the development of highly functional alternatively activated macrophages. In contrast IL-21 had no effect on the production of TGF- $\beta 1$ by macrophages. Thus, the profibrotic cytokine TGF- $\beta 1$ did not appear to be involved, which would be consistent with previous studies that have investigated the role TGF- $\beta 1$ in schistosomiasis (51). Instead, we found that IL-21 significantly increased IL-4R $\alpha$ and IL-13R $\alpha 1$ expression in bone marrow-derived macrophages and decreased the production of the sIL-13 decoy receptor in vivo, which likely explains their heightened sensitivity to IL-4 and IL-13. As such, these data compliment our in vivo studies with IL-21 $\mathrm{R}^{-/}$mice and suggest that an important function of IL-21R signaling is to enhance the development of alternatively activated macrophages, which have been implicated in the mechanism of fibrosis $(46,52)$. Moreover, because alternatively activated macrophages have been shown previously to amplify $\mathrm{CD} 4^{+} \mathrm{Th} 2$ cell differentiation (53), these data may also explain the overall reduction in helminth-induced Th2 activity in IL-21 $\mathrm{R}^{-/-}$mice.

In human schistosomiasis, the development of fibrotic liver pathology is the principle cause of chronic morbidity and mortality $(18,54)$. Because the Th2 cytokine response is known to play an important role in collagen deposition (17), in a final series of experiments we examined the influence of the IL-21R on the progression of hepatic fibrosis. Notably, development of fibrosis decreased significantly in the IL-21 $\mathrm{R}^{-/-}$mice, with the knockout animals displaying a greater than $50 \%$ reduction in hepatic fibrosis by week 29 after infection. Importantly, similar findings were also generated when infected WT mice were treated with sIL-21R-Fc. Thus the IL-21R was revealed as a potential new target for antifibrotic therapy. In conclusion, these studies illustrate an essential role for the IL-21R in the progression of Th2 cytokine-mediated disease. As such, the IL-21R should be added to list of important receptors that regulate Th2 immunity and macrophage polarization. 


\section{Methods}

Mice, parasite infections, and antigen preparation. Female or male C57BL/6, C57BL/6/Ai-IL-10KO/IL-4KO, and C57BL/6/Ai-IL-10KO/IL-12KO mice were obtained from Taconic (55). Breeding pairs of IL-21 $\mathrm{R}^{-/-}$mice on a C57BL/ 6 background were obtained from a breeding colony housed at Harvard School of Public Health (26). All mice were housed under specific pathogen-free conditions at the National Institutes of Health in an American Association for the Accreditation of Laboratory Animal Care-approved facility. The NIAID animal care and use committee approved all experimental procedures. S. mansoni eggs were extracted from the livers of infected mice (Biomedical Research Institute) as previously described (56). For the induction of synchronous primary pulmonary granulomas, mice were given 5,000 eggs i.v. For the induction of secondary granulomas, mice were sensitized i.p. with 5,000 live eggs and then challenged with 5,000 live eggs i.v. (25). In the infection experiments, mice were infected percutaneously via the tail with 25-30 cercariae of a Puerto Rican strain of S. mansoni (NMRI) that were obtained from infected Biomphalria glabrata snails (Biomedical Research Institute). SEA and soluble worm antigenic preparation (SWAP) were obtained from purified and homogenized S. mansoni eggs and adult parasites as previously described (57). All animals underwent perfusion at the time of sacrifice so that worm and tissue egg burdens could be determined as described previously (57). N. brasiliensis larvae (L3) were prepared as previously described (58). Mice were inoculated through s.c. injection of $500 \mathrm{~L} 3$. On day 7 after inoculation, lung tissue and mediastinal lymph nodes were collected for cytokine analysis.

Histopathology and fibrosis. The sizes of pulmonary and hepatic granulomas were determined on histological sections that were stained with Wright's Giemsa stain (Histopath of America). Around 30 granulomas per mouse were included in all analyses. A skilled pathologist evaluated the percentages of eosinophils, mast cells, and other types of cells in the same sections. The number of schistosome eggs in the liver and the gut and the collagen content of the liver, as measured by hydroxyproline levels, were determined as previously described (57). Hepatic collagen was measured as hydroxyproline by the technique of Bergman and Loxley (59) after hydrolysis of a 200 -mg portion of liver in $5 \mathrm{ml}$ of $6 \mathrm{~N} \mathrm{HCl}$ at $110^{\circ} \mathrm{C}$ for 18 hours. The increase in hepatic hydroxyproline was positively related to egg numbers in all experiments, and hepatic collagen was reported as the increase above normal liver collagen in $\mu \mathrm{mol}$ per 10,000 eggs ([infected liver collagen - normal liver collagen]/liver eggs $\times 10^{-4}$ ) or $\mu \mathrm{mol}$ per worm pair. At late chronic time points, fibrosis was reported as total liver collagen per liver. The same individual scored all histological features and had no knowledge of the experimental design.

Fluorescence-activated cell sorting analysis. Whole lungs were harvested and placed in RPMI. Tissues were disrupted by straining through a $70-\mu \mathrm{m}$ nylon mesh (BD Biosciences - Falcon). The single-cell suspensions were washed, and rbcs were lysed by incubation with buffered ammonium chloride lysis solution for 3 minutes. Lung lymphocytes were labeled with PE-Cy5labeled anti-CD4 along with Fc Block (both Abs from BD Biosciences Pharmingen) in fluorescence-activated cell sorting buffer for 15 minutes at $4^{\circ} \mathrm{C}$. After washing, the cells were analyzed on a FACScalibur flow cytometer (BD Biosciences) using Flowjo software (Tree Star Inc.).

IL-21 blocking experiments with sIL-21R-Fc. C57BL/ 6 mice ( $n=10$ per group) were infected percutaneously via the tail with 30-35 S. mansoni cercariae. Beginning on week 6 after infection, mice were treated with either mouse sIL-21R-Fc (Wyeth) or anti-Eimeria tenella murine $\operatorname{IgG}_{2 \mathrm{a}}$ control $\mathrm{Ab}$ (Wyeth). Each mouse received one 200- $\mu$ g dose via i.p. injection 3 times per week for a total of 5 weeks. Mice were sacrificed 12 weeks after infection, and hepatic fibrosis was measured by hydroxyproline assay.

Lymphocyte culture and cytokine detection using ELISA. Spleen and mesenteric lymph nodes (infection model) or lung-associated lymph nodes (pulmonary model) were removed aseptically, and single-cell suspensions were prepared as previously described (52). Cultures were incubated at $37^{\circ} \mathrm{C}$ in a humidified atmosphere of $5 \% \mathrm{CO}_{2}$. Cells were stimulated with SEA $(20 \mu \mathrm{g} / \mathrm{ml})$, SWAP $(50 \mu \mathrm{g} / \mathrm{ml})$, Con A $(1 \mu \mathrm{g} / \mathrm{ml})$, or medium alone. Supernatant fluids were harvested at 72 hours and assayed for cytokine production. IFN- $\gamma$, IL-5, and IL-10 were measured by sandwich ELISA using paired Abs (BD Biosciences - Pharmingen) as previously described (52). Cytokine levels were calculated with standard curves constructed using recombinant murine cytokines (BD Biosciences - Pharmingen). IL-13 levels were measured using murine IL-13 ELISA kits (R\&D Systems) according to the manufacturer's protocol. TGF- $\beta 1$ levels were quantified using mouse TGF- $\beta 1$ DuoSet ELISA Development system (R\&D Systems) according to the manufacturer's protocol. To avoid bovine-derived TGF- $\beta 1$ contamination, cells were washed 3 times in PBS and cultured in media containing $0.5 \%$ mouse serum.

$R N A$ isolation and purification and real-time PCR. Total RNA was extracted from lung and liver tissue samples placed individually in $1 \mathrm{ml}$ TRIzOL reagent (Invitrogen). The sample was homogenized using a tissue polytron (Omni International), and total RNA was extracted according to the recommendations of the manufacturer and further purified using RNeasy Mini Kit from QIAGEN. Individual sample RNA $(1 \mu \mathrm{g})$ was reverse transcribed using Superscript II Reverse Transcriptase (Invitrogen Corp.) and a mixture of oligo (dT) and random primers. Real-time RT-PCR was performed on an ABI PRISM 7900HT Sequence Detection System (Applied Biosystems). Relative quantities of mRNA for several genes was determined using SYBR Green PCR Master Mix (Applied Biosystems) and by the comparative threshold cycle method as described by Applied Biosystems for the ABI Prism 7700/7900HT Sequence Detection Systems. In this method, mRNA levels for each sample were normalized to hypoxanthine guanine phosphoribosyl transferase (HPRT) mRNA levels and then expressed as a relative increase or decrease compared with levels in uninfected controls. Primers were designed using Primer Express software (version 2.0; Applied Biosystems). Primers for IL-13, IL-4, IL-10, and HPRT (46); IL-13R $\alpha 2$ (32); and Ym1, FIZZ1, and AMCase (44) were published previously. Other primer sequences were as follows: IL-21, sense 5'-GCCAGATCGCCTCCTGATTA-3', antisense 5'-CATGCTCACAGTGCCCCTTT-3'; IL-21R, sense 5'-CTCCCCCCTTGAACGTGACT-3', antisense 5'-TTGCCCCTCAGCACGTAGTT-3'; IFN- $\gamma$, sense $5^{\prime}$-AGAGCCAGATTATCTCTTTCTACCTCAG$3^{\prime}$, antisense $5^{\prime}$-CCTTTTTCGCCTTGCTGTTG-3'.

Serum Ab isotype analysis. Total IgE was measured using the BD OPtEIA Mouse IgE ELISA Set (BD Biosciences - Pharmingen) according to the manufacturer's protocol. SEA-specific $\mathrm{IgG}_{1}$ and $\mathrm{IgG}_{2 \mathrm{~b}}$ isotype-specific $\mathrm{Ab}$ titers were evaluated by indirect ELISA. Immulon 4 plates were coated with $10 \mu \mathrm{g} / \mathrm{ml}$ SEA (100 $\mu \mathrm{l} /$ well) diluted in PBS, and serum samples were analyzed using serial 2-fold dilutions. Biotin-Rabbit Anti-Mouse IgG $_{1}$ (Zymed) was used at a 1:1,000 dilution. This was followed by Peroxidase Labeled Streptavidin (KPL) substrate enzyme at a 1:1,000 dilution. Second-step horseradish peroxidase-conjugated rabbit Rabbit Anti-Mouse $\operatorname{IgG}_{2 \mathrm{~b}}$ (Zymed) Ab was used at a 1:1,000 dilution. The absorbance in the wells was read at $405 \mathrm{~nm}$ using a Vmax Kinetic Microplate Reader (Molecular Devices) after adding $100 \mu \mathrm{l}$ one component ABTS Peroxidase Substrate (KPL).

Bone marrow-derived macrophages. Bone marrow was recovered from female C57BL/ 6 mice and cultured in Petri dishes $(100 \mathrm{~mm} \times 15 \mathrm{~mm})$ containing supplemented DMEM (L929 conditioned medium) for a period of 6 days. After 6 days, cells were harvested and seeded at a concentration of $0.5 \times 10^{6}$ cells/well in 24-well plates containing supplemented DMEM (10\% FBS, $2 \mathrm{mM}$ L-glutamine, $100 \mathrm{U} / \mathrm{ml}$ penicillin, and $100 \mu \mathrm{g} / \mathrm{ml}$ streptomycin). Cells were stimulated with IL-4, IL-13, and IL-21 (R\&D Systems) for a period of 20 hours. In some assays, cells were pretreated with IL-21. Cells were lysed and RNA was purified using the RNA Cleanup procedure with the RNeasy kits (QIAGEN). 
Arginase activity assay. Bone marrow-derived macrophages were plated at $6 \times 10^{5}$ cells/well in 96-well tissue culture plates and stimulated with combinations of IL-4, IL-13, and IL-21. IL-21 was added 6 hours prior to IL-4 or IL-13 stimulation. Following stimulation, cells were washed with PBS and lysed with $0.1 \%$ Triton X-100 containing Protease Inhibitor (Roche Diagnostics). Lysates were transferred into a 96-well PCR plate and incubated with $10 \mathrm{mM} \mathrm{MnCl}_{2}$ and $50 \mathrm{mM}$ Tris $\mathrm{HCl}$ (pH 7.5) to activate enzyme for 10 minutes at $55^{\circ} \mathrm{C}$. After enzyme activation, $25 \mu \mathrm{l}$ lysate was removed and added to $25 \mu \mathrm{l}$ $1 \mathrm{M}$ arginine ( $\mathrm{pH}$ 9.7) in a new PCR plate and incubated for 20 hours at $37^{\circ} \mathrm{C}$. $5 \mu$ l of each sample was added in duplicate to a 96-well ELISA plate along with $5 \mu \mathrm{l}$ of each standard, diluted in same assay conditions, starting at $100 \mathrm{mg} / \mathrm{dl}$. Urea determination reagent from BioAssay Systems Quantichrom Urea Assay Kit was used according to the manufacturer's protocol.

Statistics. Hepatic fibrosis (adjusted for egg number) decreases with increasing intensity of infection (worm pairs). Therefore, these variables were compared by analysis of covariance, using the logarithm of total liver eggs as the covariate and the logarithm of hydroxyproline content per egg. Variables that did not change with infection intensity were compared by 1-way ANOVA or 2-tailed Student's $t$ test (57). Changes in cytokine mRNA expression and granuloma size were evaluated using ANOVA. Differences were considered significant at ${ }^{*} P<0.05,{ }^{*} P<0.01$, and ${ }^{* * *} P<0.001$.

\section{Acknowledgments}

The authors thank Fred Lewis (Biomedical Research Institute, Rockville, Maryland, USA) for providing the parasite materials. We also thank Keith Olson, Sandy White, and the animal care staff at the NIH for technical assistance. Finally, we appreciate the helpful discussions, comments, and suggestions provided by Margaret Mentink-Kane, Rachael Reiman, Mark Wilson, and Eldad Elnekave. The Intramural Research Program of the NIH, NIAID, supported this research.

Received for publication December 20, 2005, and accepted in revised form May 2, 2006.

Address correspondence to: Thomas A. Wynn, Immunopathogenesis Section, Laboratory of Parasitic Diseases, National Institute of Allergy and Infectious Diseases, National Institutes of Health, Building 50, Room 6154, MSC 8003, Bethesda, Maryland 20892, USA. Phone: (301) 496-4758; Fax: (301) 480-5025; E-mail: twynn@niaid.nih.gov.

John Pesce and Mallika Kaviratne contributed equally to this work.
1. Parrish-Novak, J., et al. 2000. Interleukin 21 and its receptor are involved in NK cell expansion and regulation of lymphocyte function. Nature. 408:57-63.

2. Ozaki, K., Kikly, K., Michalovich, D., Young, P.R., and Leonard, W.J. 2000. Cloning of a type I cytokine receptor most related to the IL-2 receptor beta chain. Proc. Natl. Acad. Sci. U. S. A. 97:11439-11444.

3. Sivakumar, P.V., Foster, D.C., and Clegg, C.H. 2004. Interleukin-21 is a T-helper cytokine that regulates humoral immunity and cell-mediated anti-tumour responses. Immunology. 112:177-182.

4. Habib, T., Nelson, A., and Kaushansky, K. 2003. IL-21: a novel IL-2-family lymphokine that modulates B, $\mathrm{T}$, and natural killer cell responses. J. Allergy Clin. Immunol. 112:1033-1045.

5. Vosshenrich, C.A., and Di Santo, J.P. 2001 Cytokines: IL-21 joins the gamma(c)-dependent network? Curr. Biol. 11:R175-R177.

6. Ma, H.L., et al. 2003. IL-21 activates both innate and adaptive immunity to generate potent antitumor responses that require perforin but are independent of IFN-gamma. J. Immunol. 171:608-615.

7. Kishida, T., et al. 2003. Interleukin (IL)-21 and IL-15 genetic transfer synergistically augments therapeutic antitumor immunity and promotes regression of metastatic lymphoma. Mol. Ther. 8:552-558.

8. Di Carlo, E., et al. 2004. IL-21 induces tumor rejection by specific CTL and IFN-gamma-dependent CXC chemokines in syngeneic mice. J. Immunol. 172:1540-1547.

9. Collins, M., Whitters, M.J., and Young, D.A. 2003 IL-21 and IL-21 receptor: a new cytokine pathway modulates innate and adaptive immunity. Immunol. Res. 28:131-140.

10. Ozaki, K., et al. 2002. A critical role for IL-21 in regulating immunoglobulin production. Science. 298:1630-1634.

11. Suto, A., et al. 2002. Interleukin 21 prevents antigen-induced IgE production by inhibiting germ line C(epsilon) transcription of IL-4-stimulated B cells. Blood. 100:4565-4573.

12. Mehta, D.S., et al. 2003. IL-21 induces the apoptosis of resting and activated primary B cells. J. Immunol. 170:4111-4118.

13. Pene, J., et al. 2004. Cutting edge: IL-21 is a switch factor for the production of IgG1 and IgG3 by human B cells. J. Immunol. 172:5154-5157.

14. Jin, H., Carrio, R., Yu, A., and Malek, T.R. 2004. Distinct activation signals determine whether IL-21 induces B cell costimulation, growth arrest, or Bim- dependent apoptosis. J. Immunol. 173:657-665.

15. Zeng, R., et al. 2005. Synergy of IL-21 and IL-15 in regulating CD8+ $\mathrm{T}$ cell expansion and function. J. Exp. Med. 201:139-148.

16. Wurster, A.L., et al. 2002. Interleukin 21 is a Thelper (Th) cell 2 cytokine that specifically inhibits the differentiation of naive Th cells into interferon gammaproducing Th1 cells. J. Exp. Med. 196:969-977.

17. Wynn, T.A. 2004. Fibrotic disease and the $\mathrm{T}(\mathrm{H}) 1 /$ $\mathrm{T}(\mathrm{H}) 2$ paradigm. Nat. Rev. Immunol. 4:583-594.

18. Pearce, E.J., and MacDonald, A.S. 2002. The immunobiology of schistosomiasis. Nat. Rev. Immunol. 2:499-511.

19. Chiaramonte, M.G., Donaldson, D.D., Cheever, A.W., and Wynn, T.A. 1999. An IL-13 inhibitor blocks the development of hepatic fibrosis during a T-helper type 2-dominated inflammatory response. J. Clin. Invest. 104:777-785.

20. Kaplan, M.H., Whitfield, J.R., Boros, D.L., and Grusby, M.J. 1998. Th2 cells are required for the Schistosoma mansoni egg-induced granulomatous response. J. Immunol. 160:1850-1856.

21. Jankovic, D., et al. 1999. Schistosome-infected IL-4 receptor knockout (KO) mice, in contrast to IL-4 KO mice, fail to develop granulomatous pathology while maintaining the same lymphokine expression profile. J. Immunol. 163:337-342.

22. Fallon, P.G., Richardson, E.J., McKenzie, G.J., and McKenzie, A.N. 2000. Schistosome infection of transgenic mice defines distinct and contrasting pathogenic roles for IL-4 and IL-13: IL-13 is a profibrotic agent. J. Immunol. 164:2585-2591.

23. Mehta, D.S., Wurster, A.L., Weinmann, A.S., and Grusby, M.J. 2005. NFATc2 and T-bet contribute to T-helper-cell-subset-specific regulation of IL-21 expression. Proc. Natl. Acad. Sci. U. S. A. 102:2016-2021.

24. Wynn, T.A. 2003. IL-13 effector functions. Annu. Rev. Immunol. 21:425-456.

25. Wynn, T.A., Eltoum, I., Oswald, I.P., Cheever, A.W., and Sher, A. 1994. Endogenous interleukin 12 (IL-12) regulates granuloma formation induced by eggs of Schistosoma mansoni and exogenous IL-12 both inhibits and prophylactically immunizes against egg pathology. J. Exp. Med. 179:1551-1561.

26. Kasaian, M.T., et al. 2002. IL-21 limits NK cell responses and promotes antigen-specific $\mathrm{T}$ cell activation: a mediator of the transition from innate to adaptive immunity. Immunity. 16:559-569.

27. Mehta, D.S., Wurster, A.L., and Grusby, M.J. 2004.
Biology of IL-21 and the IL-21 receptor. Immunol. Rev. 202:84-95.

28. Wynn, T.A., et al. 1993. Analysis of cytokine mRNA expression during primary granuloma formation induced by eggs of Schistosoma mansoni. J. Immunol. 151:1430-1440.

29. Vella, A.T., and Pearce, E.J. 1992. CD4+ Th2 response induced by Schistosoma mansoni eggs develops rapidly, through an early, transient, Th0-like stage. J. Immunol. 148:2283-2288.

30. Nair, M.G., et al. 2005. Chitinase and Fizz family members are a generalized feature of nematode infection with selective upregulation of $\mathrm{Ym} 1$ and Fizz1 by antigen-presenting cells. Infect. Immun. 73:385-394.

31. Zhu, Z., et al. 2004. Acidic mammalian chitinase in asthmatic Th2 inflammation and IL-13 pathway activation. Science. 304:1678-1682.

32. Chiaramonte, M.G., et al. 2003. Regulation and function of the interleukin 13 receptor alpha 2 during a $\mathrm{T}$ helper cell type 2-dominant immune response. J. Exp. Med. 197:687-701.

33. Gordon, S. 2003. Alternative activation of macrophages. Nat. Rev. Immunol. 3:23-35.

34. Urban, J.F., Jr., et al. 1993. IFN inhibits inflammatory responses and protective immunity in mice infected with the nematode parasite, Nippostrongylus brasiliensis. J. Immunol. 151:7086-7094.

35. Kunkel, S.L., Chensue, S.W., Lukacs, N., and Hogaboam, C. 2003. Cytokine phenotypes serve as a paradigms for experimental immune-mediated lung diseases and remodeling. Am. J. Respir. Cell Mol. Biol. 29:S63-S66.

36. Mentink-Kane, M.M., et al. 2004. IL-13 receptor alpha 2 down-modulates granulomatous inflammation and prolongs host survival in schistosomiasis. Proc. Natl. Acad. Sci. U. S. A. 101:586-590.

37. Wood, N., et al. 2003. Enhanced interleukin (IL)-13 responses in mice lacking IL-13 receptor alpha 2. J. Exp. Med. 197:703-709.

38. Jakubzick, C., et al. 2003. Impact of interleukin-13 responsiveness on the synthetic and proliferative properties of Th1- and Th2-type pulmonary granuloma fibroblasts. Am. J. Pathol. 162:1475-1486.

39. Zheng, T., et al. 2003. Cytokine regulation of IL13Ralpha2 and IL-13Ralpha1 in vivo and in vitro. J. Allergy Clin. Immunol. 111:720-728.

40. Morimoto, M., et al. 2006. Functional importance of regional differences in localized gene expression of receptors for IL-13 in murine gut. J. Immunol. 
176:342-348.

41. Guo, L., Johnson, R.S., and Schuh, J.C. 2000. Biochemical characterization of endogenously formed eosinophilic crystals in the lungs of mice. J. Biol. Chem. 275:8032-8037.

42. Liu, T., et al. 2004. Regulation of found in inflammatory zone 1 expression in bleomycin-induced lung fibrosis: role of IL-4/IL-13 and mediation via STAT-6. J. Immunol. 173:3425-3431.

43. Hoffmann, K.F., Cheever, A.W., and Wynn, T.A. 2000. IL-10 and the dangers of immune polarization: excessive type 1 and type 2 cytokine responses induce distinct forms of lethal immunopathology in murine schistosomiasis. J. Immunol. 164:6406-6416.

44. Sandler, N.G., Mentink-Kane, M.M., Cheever, A.W., and Wynn, T.A. 2003. Global gene expression profiles during acute pathogen-induced pulmonary inflammation reveal divergent roles for Th1 and Th 2 responses in tissue repair. J. Immunol. 171:3655-3667.

45. Mantovani, A., Sica, A., and Locati, M. 2005. Macrophage polarization comes of age. Immunity. 23:344-346.

46. Hesse, M., et al. 2001. Differential regulation of nitric oxide synthase- 2 and Arginase- 1 by type 1/ type 2 cytokines in vivo: granulomatous pathology is shaped by the pattern of L-arginine metabolism. J. Immunol. 167:6533-6544.

47. Herbert, D.R., et al. 2004. Alternative macrophage activation is essential for survival during schistosomiasis and downmodulates $T$ helper 1 responses and immunopathology. Immunity. 20:623-635.

48. Lee, C.G., et al. 2001. Interleukin-13 induces tissue fibrosis by selectively stimulating and activating transforming growth factor beta(1). J. Exp. Med. 194:809-821.

49. Fichtner-Feigl, S., Strober, W., Kawakami, K., Puri, R.K., and Kitani, A. 2006. IL-13 signaling through the IL-13alpha2 receptor is involved in induction of TGF-beta1 production and fibrosis. Nat. Med. 12:99-106.

50. Munder, M., Eichmann, K., and Modolell, M. 1998. Alternative metabolic states in murine macrophages reflected by the nitric oxide synthase/arginase balance: competitive regulation by $\mathrm{CD} 4+\mathrm{T}$ cells correlates with Th1/Th2 phenotype. J. Immunol. 160:5347-5354.

51. Kaviratne, M., et al. 2004. IL-13 activates a mechanism of tissue fibrosis that is completely TGF-beta independent. J. Immunol. 173:4020-4029.

52. Hesse, M., Cheever, A.W., Jankovic, D., and Wynn, T.A. 2000. NOS- 2 mediates the protective antiinflammatory and antifibrotic effects of the Th1 inducing adjuvant, IL-12, in a Th2 model of granulomatous disease. Am. J. Pathol. 157:945-955.

53. Bonecchi, R., et al. 1998. Divergent effects of interleukin-4 and interferon-gamma on macrophagederived chemokine production: an amplification circuit of polarized T helper 2 responses. Blood. 92:2668-2671.

54. Wynn, T.A., Thompson, R.W., Cheever, A.W., and Mentink-Kane, M.M. 2004. Immunopathogenesis of schistosomiasis. Immunol. Rev. 201:156-167.

55. Hoffmann, K.F., James, S.L., Cheever, A.W., and Wynn, T.A. 1999. Studies with double cytokinedeficient mice reveal that highly polarized Th1and Th2-type cytokine and antibody responses contribute equally to vaccine-induced immunity to Schistosoma mansoni. J. Immunol. 163:927-938.

56. Wynn, T.A., et al. 1995. An IL-12-based vaccination method for preventing fibrosis induced by schistosome infection. Nature. 376:594-596.

57. Cheever, A.W. et al. 1994. Anti-IL-4 treatment of Schistosoma mansoni-infected mice inhibits development of T cells and non-B, non- $\mathrm{T}$ cells expressing Th2 cytokines while decreasing egg-induced hepatic fibrosis. J. Immunol. 153:753-759.

58. Katona, I.M., Urban, J.F., Jr., Scher, I., Kanellopoulos-Langevin, C., and Finkelman, F.D. 1983. Induction of an IgE response in mice by Nippostrongylus brasiliensis: characterization of lymphoid cells with intracytoplasmic or surface IgE. J. Immunol. 130:350-356.

59. Bergman, I., and Loxley, R. 1963. Two improved and simplified methods for the spectrophotometric determination of hydroxyproline. Anal. Biochem. 35:1961-1965. 